(C) 2013

Скакалина Е. В., кандидат технических наук

Полтавский национальный технический университет им. Юрия Кондратюка

\title{
ПОДХОД К РЕШЕНИЮ ЗАДАЧИ ОПТИМИЗАЦИИ ЛОГИСТИКИ АГРОХОЛДИНГА
}

\section{Рецензент - доктор технических наук, профессор А. Л. Ляхов}

У роботі наведено короткий аналіз використання інформаційних технологій в аграрному напрямі. Вказується на можливість удосконалення управління процесом реалізації логістики великих агрохолдингів за рахунок використання ефективного методу побудови оптимальних рішень для узагальнень задачі про призначення. Представлений новий клас дискретних оптимізаційних задач. Звертається увага на інтенсивний розвиток логістики у зарубіжних країнах на основі використання сучасних комп'ютерних технологій.

Ключевые слова: агрохолдинг, логистика, информационные технологии, оптимальное распределение ресурсов, модели и методы оптимального назначения и упорядочения.

Постановка проблемы. Единственным сектором экономики Украины, который продемонстрировал рост в разгар финансово-экономического кризиса, было сельское хозяйство. За последние пять лет инвестиционная привлекательность украинского аграрного сектора возросла в разы. Оплотом роста и ключевым объектом внимания западных инвесторов выступают крупные аграрные холдинги Украины, прибыльность которых в два раза выше, чем у аналогичных структур в Западной Европе и в семь раз чем в США.

Если в начале 2000-х в центре внимания инвестиционных банков и хедж-фондов пребывали такие сектора, как телекоммуникация, строительство, металлургия, рынок драгоценных металлов, то сейчас все увереннее выходит на первый план аграрный сектор. Только в США в период 2007-2011 гг. было инвестировано $\$ 1,3$ млрд в сельхозземли. Главной причиной проявления такой «симпатии» являются динамичный рост цен на продовольственные товары и спрос на биоэтанол.

По данным Продовольственной и сельскохозяйственной организации ООН (Food and Agriculture Organization, FAO), к 2050 году население планеты возрастет до 9,1 млрд человек (по сравнению с нынешней численностью 7 млрд). Таким образом, в последующие 37 лет прирост населения составит 30 \%. Последние оценки FAO указывают на то, что для удовлетворения потребностей населения в пище объем сельскохозяйственного производ-ства за тот же период должен будет возрасти на $70 \%$.

В то же время рост сельскохозяйственного производства, согласно прогнозам, будет, наоборот, замедляться и составит в ближайшее десятилетие около $2 \%$ в год. Учитывая же, что на нашу страну приходится $75 \%$ европейских и $25 \%$ мировых запасов черноземов, украинские агрохолдинги априори получают конкурентное преимущество.

Для сохранения конкурентоспособности украинских агрохолдингов ключевую роль будут играть их системы управления (структурный капитал) и трудовые ресурсы. Одним из способов усиления и усложнения для имитации структурного капитала и повышения эффективности может выступить переход от функциональной к процессуальной организационной культуре. Система управления агрохолдингом на основе функциональной модели использует разделение труда и производственной специализации, а на основе процессно-ролевой модели - выделение бизнес-процессов. Преимущества этой модели включают: способность гибкой реакции на изменения внешних условий, постоянное совершенствование, акцент на командной работе и скорость трансформации бизнес-процессов. Так, новые технологии, внедряемые одним предприятием, могут быть заимствованы другим через некую координирующую структуру. Процессный подход на уровне холдинга также позволяет более полно реализовать преимущества межхозяйственной специализации и снизить риски.

Анализ основных исследований и публикаций по данной проблеме. Большинство экспертов в сфере стратегии признают, что интеллектуальный капитал более ценен, нежели материальный. В отличие от материальных ресурсов, которые могут обесцениваться в процессе использования, знание увеличивается, если оно используется, и обесценивается, если не используется [3].

Именно благодаря способности создавать знания компании имеют возможность достигать устойчивого конкурентного преимущества. Поэтому для агрохолдингов необходимо развивать стратегические компетенции своих сотрудников. Люди являются ключевыми действующими лица- 


\section{TEХНIЧНІ НАУКИ}

ми в бизнесе: все продукты, активы и отношения это результаты человеческой деятельности, продолжительность и качество которых также зависят от нee.

На сегодняшний день отечественные агрохолдинги - одна из самых эффективных форм ведения агробизнеса, обеспечивающих наибольший прирост сельскохозяйственного производства Украины. Данные холдинговые структуры, инвестируя в сельское хозяйство, стремятся формировать полный цикл «производство - переработка - продажа», внедрять современные технологии, обновлять парк сельхозтехники и обеспечивать активное присутствие на рынках сбыта.

Украинские агрохолдинги демонстрируют высокую прибыльность за счет снижения себестоимости сельскохозяйственной продукции. Но факторы, на которых основана данная стратегия, не носят устойчивого характера.

Для получения высокой ренты на протяжении более длительного периода времени украинским агрохолдингам необходимо сфокусироваться на эффективном использовании не только материальных, но и нематериальных ресурсов (человеческого и структурного капитала).

Рыночные условия сельскохозяйственного производства постоянно меняются и совершенно очевидно, что для его успешного и прибыльного функционирования необходимо, кроме применения современной техники, внедрение передовых научных разработок и инновационных технологий. Качество функционирования современного производства во многом определяется решениями, принимаемыми на этапах календарного планирования и оперативного управления. Наряду с улучшением качества плановых решений все более жесткими становятся требования к сокращению сроков их выработки, повышению оперативности и гибкости управления.

Многие практические ситуации приводят к необходимости изучения многостадийных обслуживающих систем, т. е. таких систем, в которых процесс обслуживания каждого требования состоит из нескольких последовательных стадий, на каждой из которых это требование обслуживается тем или иным прибором или совокупностью приборов. Так, посев зерновых включает несколько последовательных операций, каждая из которых выполняется некоторым МТА (погрузка семян, погрузка минеральных удобрений, транспортировка семян и минеральных удобрений, непосредственно посев).

В наиболее общей формулировке задачи составления расписаний состоят в следующем. С помощью некоторого множества ресурсов должна быть выполнена некоторая фиксированная система за- даний. Цель заключается в том, чтобы при заданных свойствах заданий и ресурсов, и наложенных на них ограничениях найти эффективный алгоритм упорядочения заданий, оптимизирующий или стремящийся оптимизировать желаемую меру эффективности. В качестве основных мер эффективности рассматриваются, как правило, длина расписания и среднее время пребывания заданий в системе. Модели этих задач являются детерминированными, то есть вся информация, на основе которой принимаются решения об упорядочении, известна заранее. Задания и все данные о них предполагаются известными в начальный момент времени $\mathrm{t}=0$.

Модель процесса упорядочения представляется в виде совокупности моделей, описывающих ресурсы, систему заданий, ограничения предшествования и меры оценки расписаний.

В большинстве моделей технические ресурсы состоят из набора процессоров $\mathrm{N}^{*}=\{\mathrm{n} 1, \ldots \mathrm{n} \mu\}$. В зависимости от особенностей задачи, они являются либо идентичными, либо одинаковыми только по функциональным возможностям, но разными по быстродействию, либо разными как по возможностям, так и по быстродействию [2].

Ограничения при составлении расписаний в общем виде могут быть:

1. Составление расписаний без прерываний работа раз начавшись, не может быть прервана, т. е. выполнение работы всегда доходит до конца.

2. Составление расписаний с прерываниями разрешается прерывать работу, выполняемую MTA; при этом полагаем, что общее время, требуемое для выполнения работы, остается неизменным, и при прерываниях отсутствуют потери времени обслуживания.

3. Составление расписаний с помощью списка предполагается, что вначале готовится упорядоченный список работ (список приоритетов).

Последовательность, в соответствии с которой работы назначаются на МТА, составляется путем многократного просмотра списка. В частности, если появляется освободившийся МТА, то список начинает просматриваться сначала и просматривается до тех пор, пока не найдется первая невыполненная работа i, которая готова к выполнению. Работа считается готовой к выполнению на данном МТА, если выполнение всех предшественников i завершено и имеющегося количества ресурсов $\mathrm{N}$ достаточно для того, чтобы обеспечить её завершение в допустимые сроки. Это задание назначается для выполнения на свободный МТА. Мы предполагаем, что каждый просмотр осуществляется мгновенно. Кроме то- 


\section{ТЕХНІЧНІ НАУКИ}

го, если одновременно два или более МТА оказываются свободными, мы предполагаем, что сначала назначается работа на N1, затем на N2, затем на N3 и так далее. При этом прерывания не рассматриваются формально; расписание может быть определено как некоторое отображение, которое, в общем случае, сопоставляет каждой работе последовательно из одного или более непересекающихся временных интервалов, лежащих в $[0, \infty]$ с выполнением следующих требований:

a) на каждый интервал назначается один MTA;

б) сумма длин интервалов равна времени выполнения работы, с учетом разной скорости их выполнения;

в) никакие два интервала, относящиеся к разным работам, назначенным на один и тот же MTA, не перекрываются;

г) учтены ограничения на порядок выполнения работ и использование дополнительных видов ресурсов;

д) на отрезке $[0, \max \{f i\}]$ не существует интервалов, на которых ни один из МТА не был бы наделен какой-либо работой (т. е. в расписании не разрешается иметь МТА свободными, если остаются незавершенными работы). В расписаниях без прерываний для каждой работы отводится ровно один интервал, а при составлении расписаний с помощью списка еще накладывается требование, чтобы ни один МТА не мог быть свободным, если имеются готовые к выполнению работы, которые могут быть на него назначены.

В основном рассматривают четыре основных показателя эффективности [1], длину расписания или максимальное время завершения $\Delta \mathrm{t}(\Phi)=\max \{\mathrm{fi}(\Phi)\}$ при $1 \leq \mathrm{i} \leq \mathrm{n}$, среднее взвешенное время завершения или прохождения $\Delta \mathrm{t}(\Phi)=$ 1 $\bar{n} \sum \operatorname{sifi}(\Phi)$ при $\mathrm{i}=1, \mathrm{n}$.

Основная проблема заключается в нахождении эффективных алгоритмов, позволяющих находить среди всех расписаний такие, для которых эти величины достигают минимума (максимума).

Особое внимание следует обратить на один из способов приближенного решения задач теории расписаний, сущность которого заключается в следующем. Сначала решается задача нахождения допустимого расписания для технологических средств, как основных, так и резервных.

Результатом решения этой задачи являются интервалы директивного времени, в которые выполняются отдельные работы без потерь уро- жая. Эти интервалы являются основанием для последующего точного распределения МТА по работам с учетом их соответствия сезонным условиям и ресурсосбережения. Решение задачи завершается построением сезонного расписания, в соответствие с выбранным экономическим критерием, учитывающим как основне, так и резервные технико-технологические средства.

Декомпозиция исходной задачи на две более простые закладывается в основу метода упорядочения параллельных производственных процессов растениеводства, функционирующих в различных погодных условиях.

Первая задача решается на основании учета климатических, погодных и агробиологических особенностей процессов в сроках использования технических средств - составления допустимого расписания.

Допустимые расписания параллельных производственных процессов составляются с учетом потребностей каждой работы к условиям её проведения. Допустимые расписания образуют форму, которая должна уточняться содержанием - ресурсными, организационными и экономическими возможностями производства. В этом отношении они имеют самостоятельное значение при любом разнообразии технической оснащенности и обеспеченности другими ресурсами.

Цель исследований: обобщение, развитие и усовершенствование моделей последовательнопараллельного упорядочения и назначения работ, разработка вычислительной схемы решения класса задач оптимального назначения и параллельного выполнения работ, включая задачи эффективной организации логистических процессов.

Результаты исследований. Рассмотрим систему из m параллельных неидентичных машин, предназначенных для выполнения $\mathrm{n}$ одноэтапных работ, $\mathrm{n}>\mathrm{m}$. Пусть $\left[\beta_{\mathrm{ij}}\right]_{\mathrm{n \times m}}$ обозначает матрицу, в которой есть $\beta_{\mathrm{ij}}$ время выполнения работы ${ }^{\mathrm{j}}$ на машине $^{\text {i }}$ i $=1, \mathrm{~m}, \mathrm{j}=1, \mathrm{n}$

Определим расписание выполнения $\mathrm{n}$ работ на $\mathrm{m}$ машинах как последовательность $\Pi=\left(\pi_{1}, \pi_{2}, \ldots \pi_{\mathrm{i}}, \ldots \pi_{\mathrm{m}}\right)$, в которой перестановка $\pi_{\mathrm{i}}$ задаёт порядок работ, назначенных на машину $\mathbf{i}$ Пусть в расписании П работа ', выполняемая машиной ${ }^{\mathbf{i}}$, завершается в момент времени $\mathbf{f}_{\mathrm{ij}}(\boldsymbol{\Pi})$.

Для расписания П найдём суммарное время выполнения $\mathrm{n}$ работ на $\mathrm{m}$ параллельных машинах, 
равное $\operatorname{mwft}(\Pi)=\sum_{\mathrm{j}=\mathrm{I}}^{\mathrm{n}} \sum_{\mathrm{i}=\mathrm{I}}^{\mathrm{m}} \mathrm{f}_{\mathrm{ij}}(\Pi)$.

Требуется построить расписание $\Pi^{*}$ с минимальным суммарным временем завершения работ.

В [5] показано, что искомое расписание П* находится в результате выполнения процедуры определения потока минимальной стоимости в транспортной сети ${ }^{\mathrm{N}}$, которая строится для мат-

$$
\mathrm{C}=\left[\begin{array}{c}
{\left[\beta_{\mathrm{ij}}\right]} \\
{\left[2 \beta_{\mathrm{ij}}\right]} \\
\cdots \cdots \\
{\left[\mathrm{n} \beta_{\mathrm{ij}}\right]}
\end{array}\right]
$$

рицы

$\left[\mathrm{r} \beta_{\mathrm{ij}}\right]$ обозначает матрицу, образованную из $\left[\beta_{i j}\right.$ $\mathrm{m} \times \mathrm{n}$ умножением каждого её элемента на $\mathbf{r}$.

Рассмотрим, как, применяя алгоритм решения задачи построения всех расписаний с минимальным суммарным временем завершения работ, выполняемых на неидентичных машинах, можно построить за полиномиальное время все расписания $\Pi^{*}$ с минимальным суммарным временем завершения работ. Определим матрицу

$\mathrm{B}=\mathrm{CT}=\left[\left[\beta_{\mathrm{ij}}\right]^{\mathrm{T}},\left[2 \beta_{i j}\right]^{\mathrm{T}}, \ldots,\left[\mathrm{r} \beta_{\mathrm{ij}}\right]^{\mathrm{T}}, \ldots,\left[\mathrm{n} \beta_{\mathrm{ij}}\right]^{\mathrm{T}}\right]$ и обозначаем её элементы $\beta_{\mathrm{ij}}^{0}$, $\mathrm{i}=\overline{\mathrm{l}, \mathrm{n}}, \mathrm{j}=\overline{\mathrm{l}, \mathrm{m}^{\prime}}, \mathrm{m}^{\prime}=\mathrm{nm}$

Пусть матрица В является входом алгоритма решения задачи, а перестановка $\pi^{*}=\left(\beta_{\pi^{*}(1)}, \beta_{\pi^{*}(2)}, \beta_{\pi^{*}\left(m^{\prime}\right)}\right)$ есть одно из её оптимальных решений.

Тогда значение $\beta_{\pi^{*}(1)}=\beta_{\mathrm{pq}}^{0}, \mathrm{p} \leq \mathrm{n}$, в задаче построения расписания П* вносит вклад $\operatorname{mwft}\left(\Pi^{*}\right)$ при условии, что работа р выполняется последней на соответствующей машине. Значение $\beta_{\pi^{*}(\mathrm{k})}=\beta_{\mathrm{pq}}^{0}=2 \beta_{\mathrm{rs}}, \mathrm{r} \leq \mathrm{n}$ представляет собой слагаемое в $\mathrm{mwft}\left(\Pi^{*}\right)$ в предложении, что работа $r$ завершается предпоследней на соответствующей машине, и так далее.

Таким образом, решению $\pi^{*}$ соответствуют решение П*, и алгоритм решения задачи корректно находит все решения задачи минимизации суммарного времени выполнения работ.

В общем случае допустимое расписание $\Pi=\left(\pi_{1}, \pi_{2}, \ldots \pi_{\mathrm{i}}, \ldots \pi_{\mathrm{m}}\right)$ задачи, в котором блок $\pi_{\mathrm{i}}$ предоставляет собой последовательность эле- ментов i-й строки матрицы $\left[\beta_{\mathrm{ij}}\right]_{\mathrm{m \times n}}$, строится из допустимого решения $\pi$ задачи с помощью следующей процедуры:

1. Входные данные задачи: $\left[\beta_{\mathrm{ij}}\right]_{\mathrm{m \times n}}$ - матрица, в которой элемент $\beta_{\mathrm{ij}}$ равен времени выполнения работы j на машине i; $\pi$ - допустимое решение задачи, представленное перестановкой $\mathrm{n}$ строк матрицы $\mathrm{B}=\mathrm{C}^{\mathrm{t}}\left[\beta_{\mathrm{rl}}^{0}\right]_{\mathrm{n \times m}}, \mathrm{m}^{\prime}=\mathrm{nm}$; если $\beta_{\mathrm{rl}}^{0} \in \pi_{\text {и }} \beta_{\mathrm{pq}}^{0} \in \pi$, то $\mathrm{r} \neq \mathrm{p}, \mathrm{l} \neq \mathrm{q}, \mathrm{k}=\mathrm{l}$.

2. Пока $\mathrm{k} \leq \mathrm{n}$, определить индекс і блока $\pi_{\mathrm{i}}$, которому принадлежит элемент ${ }^{\mathrm{ij}}$, соответствующий элементу $\beta_{\mathrm{kl}}^{0} \in \pi$; индекс і находится с помощью соотношений

$\beta_{\mathrm{kl}}^{0}=r \beta_{\mathrm{ij}}, \mathrm{k}=\mathrm{j}, \mathrm{r}=\overline{\mathrm{l}, \mathrm{n}} \mathrm{l}=\mathrm{rm}+\mathrm{i}^{\prime}, 0 \leq \mathrm{i}^{\prime} \leq \mathrm{m}$; при этом $\mathrm{i}=\mathrm{m}, \beta_{\mathrm{ij}} \in \pi_{\mathrm{m}}$, если $\mathrm{i}^{\prime}=0$, и $\mathrm{i}=\mathrm{i}^{\prime}$, $\beta_{\mathrm{ij}} \in \pi_{\mathrm{i}}$ в противном случае; $\mathrm{k}=\mathrm{k}+\mathrm{l}$.

3. Упорядочить элементы каждого набора, полученного на шаге 2 , по убыванию значения коэффициентов $r$, связывающих параметры $\beta_{\mathrm{kl}}^{0}$ и $\beta_{\mathrm{ij}}$; в результате получим все ${ }^{\mathrm{m}}$ блоков допустимого решения $\Pi=\left(\pi_{1}, \pi_{2}, \ldots \pi_{1}, \ldots \pi_{\mathrm{m}}\right)$ задачи.

Следует отметить, что в решении $\Pi=\left(\pi_{1}, \pi_{2}, \ldots \pi_{\mathrm{i}}, \ldots \pi_{\mathrm{m}}\right)$ допустимы пустые блоки.

Обратимся к примеру, иллюстрирующему связь задачи построения расписаний в постановке с задачей нахождения всех оптимальных решений.

$$
\text { Пусть }\left[\beta_{\mathrm{ij}}\right]_{2 \times 5}=\left[\begin{array}{lllll}
4 & 6 & 5 & 2 & 3 \\
2 & 3 & 5 & 1 & 2
\end{array}\right] \text {. }
$$

Расписание П является последовательностью $\left(\pi_{1}, \pi_{2}\right)$, содержащей две перестановки: перестановка $\pi_{1}$ задаёт порядок выполнения работ, назначенных на первую машину, а $\pi_{2}$ указывает, какие работы и в какой очерёдности выполняются на второй машине.

Чтобы применить алгоритм решения задачи для нахождения всех оптимальных расписаний $\Pi^{*}$ задачи , образуем из $\left[\beta_{\mathrm{ij}}\right]_{2 \times 6}$ матрицу 


$\left[\beta_{\mathrm{ij}}^{0}\right]_{5 \times 10}=\left[\begin{array}{cccccccccc}4 & 2 & 8 & 4 & 12 & 6 & 16 & 8 & 20 & 10 \\ 6 & 3 & 12 & 6 & 18 & 9 & 24 & 12 & 30 & 15 \\ 5 & 5 & 10 & 10 & 15 & 15 & 20 & 20 & 25 & 25 \\ 2 & 1 & 4 & 2 & 6 & 3 & 8 & 4 & 10 & 5 \\ 3 & 2 & 6 & 4 & 9 & 6 & 12 & 8 & 15 & 10\end{array}\right]$

Рассмотрим допустимое решение:

$\pi=\left(\beta_{11}^{0}, \beta_{24}^{0}, \beta_{32}^{0}, \beta_{46}^{0}, \beta_{58}^{0}\right), \beta_{11}^{0}=4, \beta_{24}^{0}=6, \beta_{32}^{0}=5, \beta_{46=3}^{0}, \beta_{58}^{0}=8$. Решению $\pi$ соответствует расписание $\Pi=\left(\pi_{1}, \pi_{2}\right)$ где $\pi_{1}=\left(\beta_{11}\right)$, a $\pi_{2}$ определяется перестановкой $\left(\beta_{25}, \beta_{24}, \beta_{22}, \beta_{23}\right)$. Таким образом для расписания П, представленного на рис. 1 , сумма моментов окончания работ, длительности выполнения которых определяются из матрицы $\left[\beta_{\mathrm{ij}}\right]_{2 \times 5}$, оказывается равной $\operatorname{mwft}(\Pi)=\left(\beta_{25}+\beta_{25}+\beta_{24}+\beta_{25}+\beta_{24}+\beta_{22}+\beta_{25}+\beta_{24}+\beta_{22}+\beta_{23}+\beta_{11}\right)=15$

\begin{tabular}{|l|l|l|l|l|}
\hline$\beta_{11}=4$ & $\beta_{25}=2$ & $\beta_{24}=1$ & $\beta_{22}=3$ & $\beta_{23}=5$ \\
\hline
\end{tabular}

\section{Puc. Расписание II}

Приведенные рассуждения являются обоснованием корректного применения схемы минимизации функции $\mathrm{F} 0(\pi)$ для решения обобщения задачи минимизации суммарного времени выполнения работ на неидентичных машинах, состоящего в построении множества всех расписаний с наименьшей суммой моментов завершения заданной совокупности работ. Следующий алгоритм находит все расписания П* за полиномиальное время [4]:

p. $1\left[\beta_{\mathrm{ij}}\right]_{\mathrm{m \times n}-\text { входные данные задачи, где }} \beta_{\mathrm{ij}}$ - время выполнения работы j на машине $\mathrm{i}$.

p. 2 Из $\left[\beta_{\mathrm{ij}}\right]_{\mathrm{m} \times \mathrm{n}}$ образовать матрицу $\mathrm{B}=\left[\left[\beta_{\mathrm{ij}}\right]^{\mathrm{T}},\left[2 \beta_{\mathrm{ij}}\right]^{\mathrm{T}}, \ldots,\left[\mathrm{r} \beta_{\mathrm{ij}}\right]^{\mathrm{T}}, \ldots,\left[\mathrm{n} \beta_{\mathrm{ij}}\right]^{\mathrm{T}}\right]_{\text {c }} \mathrm{n}$ строками и $\mathrm{m}^{\prime}=\mathrm{mn}$ столбцами; $\mathrm{B}=\left[\beta_{\mathrm{ij}}^{0}\right]_{\mathrm{m \times n}}$, - исходная матрица задачи минимизации функции $\mathrm{F} 0(\pi)$.

p. 3 . Выполнить шаги $\mathrm{p} 1-\mathrm{p} 4$ алгоритма построения допустимого решения для исходных данных, представленных матрицей $\mathrm{B}=\left[\beta_{\mathrm{ij}}^{0}\right]_{\mathrm{m} \times \mathrm{n}}$.

\section{БИБЛИОГРАФИЯ}

1. Автоматизированный системно-когнитивный анализ и его применение для управления социально-экономическими системами в АПК / Е. В. Луценко, В. И. Лойко, О.А.Макаревич, Л. О. Макаревич // Политематический сетевой электронный научный журнал Кубанского государственного аграрного университета (Научный p. 4 . Построить множество всех решений $\pi^{*}$ из $\mathrm{n}$ компонент, доставляющих минимум функции $\mathrm{F} 0(\pi)$.

p. 5 . Для каждого полученного решения $\pi^{*}$ выполнить процедуру построения соответствующего оптимального расписания $\Pi^{*}=\left(\pi_{1}^{*}, \pi_{2}^{*}, \ldots, \pi_{m}^{*}\right)$

Выводы. Практика показывает, что инвестиции на создание и внедрение инновационных оптимизационных задач при управлении такими сложными объектами как агрохолдинги окупаются достаточно быстро за счет повышения использования транспортных средств. В зарубежной практике продолжается интенсивное развитие логистики на основе использования информационных технологий. Построенные модели и на их основе сформулированные важные оптимизационные задачи последовательно-параллельного упорядичения и назначения работ на неидентичные машины. На основе разработанной схемы получены эффективные алгоритмы получения решения задач построения оптимальных расписаний.

журнал КубГАУ). - Краснодар : КубГАУ, 2012. - №04(78). - С. 706-719.

2. Алесинская Т. В. Основы логистики. Функциональные области логистического управления. - Ч. 3. - Таганрог : Изд-во ТТИ ЮФУ, 2010. - 116 с.

3. Мошнянский А. А., Мошнянский А. Ф. Ин- 
формационные технологии на транспорте и в логистике // Методи та засоби управління розвитком транспортних систем : Зб. наук. праць. - Одеса : ОНМУ, 2012. - Вип.19. - С. 175-183. 4. Скакалина Е. В. Эффективное построение множества расписаний с минимальным сум- марным временем завершения работ // Радиоэлектроника и информатика. - 2001. - №3(16). - C. 44-46.

5. Теория расписаний и вычислительные машины // Под ред. Коффмана Э. Г. - М. : Наука. - 1984. - 334 с. 